\title{
MARKOV PROCESSES WITH LIPSCHITZ SEMIGROUPS
}

\author{
BY
}

RICHARD BASS

ABstract. For $f$ a function on a metric space, let

$$
\text { Lip } f=\sup _{x \neq y}|f(x)-f(y)| / d(x, y),
$$

and say that a semigroup $P_{t}$ is $\operatorname{Lipschitz}$ if $\operatorname{Lip}\left(P_{t} f\right)<e^{K t} \operatorname{Lip} f$ for all $f$, $t$, where $K$ is a constant. If one has two Lipschitz semigroups, then, with some additional assumptions, the sum of their infinitesimal generators will also generate a Lipschitz semigroup. Furthermore a sequence of uniformly Lipschitz semigroups has a subsequence which converges in the strong operator topology.

Examples of Markov processes with Lipschitz semigroups include all diffusions on the real line which are on natural scale whose speed measures satisfy mild conditions, as well as some jump processes. One thus gets Markov processes whose generators are certain integro-differential operators. One can also interpret the results as giving some smoothness conditions for the solutions of certain parabolic partial differential equations.

1. Introduction. If $f$ is a function on a metric space, let

$$
\operatorname{Lip} f=\sup _{x \neq y}|f(x)-f(y)| / d(x, y) \text {. }
$$

Say that a semigroup $P_{t}$ is Lipschitz if there is a constant $K$ such that $\operatorname{Lip}\left(P_{t} f\right) \leqslant$ $e^{K t} \operatorname{Lip} f$ for all $t$ and $f$. It is not too surprising that the Markov processes that arise out of Ito's theory of stochastic differential equations have semigroups that are Lipschitz. What may be more surprising is that any diffusion on the reals that is on natural scale and whose speed measure satisfies certain mild conditions also has Lipschitz semigroups and, in $\operatorname{fact}, \operatorname{Lip}\left(P_{t} f\right) \leqslant \operatorname{Lip} f$.

One consequence of this fact is that it can be shown that if

$$
A g(x)=a(x) g^{\prime \prime}(x)+b(x) g^{\prime}(x)+\int\left[g(y)-g(x)-g^{\prime}(x)(y-x)\right] m(x, d y)
$$

when $g$ is twice continuously differentiable, then $A$ will be the generator of a strong Markov process that has Lipschitz semigroups, provided $a$ is nonnegative, continuous, and bounded, $b$ is Lipschitz, and $m$ satisfies a Lipschitz-like condition (see §7). This is an extension of results of Skorokhod [16] and Lepeltier and Marchal [14] in that here $a$ need not be Lipschitz and the conditions on $m$ are weaker than those that they use. Komatsu [12] and Stroock [17] have results that can be applied here, but only in the case that $a$ is strictly positive; we allow $a$ to be zero.

Received by the editors November 6, 1980.

1980 Mathematics Subject Classification. Primary 60J35; Secondary $60 \mathrm{~J} 60$.

Key words and phrases. Semigroups, infinitesimal generators, jump processes, diffusions, parabolic partial differential equations. 
The fact that diffusions on the real line have Lipschitz semigroups can be given an interpretation in terms of partial differential equations. If $P_{t}$ is the semigroup of a diffusion with infinitesimal generator $A=(d / d m)(d / d x)$, where $m$ is the speed measure, then $u(t, x)=P_{t} f(x)$ is the solution of the parabolic partial differential equation $\partial u / \partial t=A u$ with initial condition $u(0, x)=f(x)$. If $A$ is written as $a(x)\left(d^{2} / d x^{2}\right)$, our $a$ need not be continuous nor strictly positive. The assertion that $P_{t}$ is Lipschitz is then an assertion about the smoothness of the solution $u$ in the spatial variable in terms of the smoothness of the initial condition $f$. We thus generalize some of the results of Fichera [7], Freidlin [8], Kostin [13], and Oleinik [15]. See also [5] and [11].

In $\$ 2$ we prove that if one has two Lipschitz semigroups, then the sum of their infinitesimal generators will also generate a Lipschitz semigroup. We also prove that given a sequence of uniformly Lipschitz semigroups, there will be a subsequence that converges in the strong operator topology. In $\S \S 3$ and 4 we prove that any diffusion on the real line that is on natural scale and whose speed measure satisfies certain mild conditions will have Lipschitz semigroups. In $\$ 5$ we consider the drift term, and in $\$ \S 6$ and 7 we extend the class of jump processes considered by Skorokhod and Lepeltier and Marchal in two different ways. In $§ 7$ we also give an example of a semigroup that is not Lipschitz.

2. Semigroups. Let $E$ be $r$-dimensional Euclidean space. Let $C_{0}$ be the continuous functions that vanish at infinity. Let $C_{0}^{2}$ be the set of functions in $C_{0}$ whose first and second order partial derivatives are also in $C_{0}$. Let $d$ be the Euclidean metric, and let $\|f\|=\sup _{x \in E}|f(x)|$.

Call a nonnegative contraction semigroup, $P_{t}$, Feller if $P_{t}: C_{0} \rightarrow C_{0}$ and if $\left\|P_{t} f-f\right\| \rightarrow 0$ as $t \rightarrow 0$ whenever $f \in C_{0}$. A standard construction gives a correspondence between strong Markov Processes $\left(Z_{t}, P^{x}\right)$ and Feller semigroups $P_{t}[3]$.

If $f \in C_{0}$, define

$$
\operatorname{Lip} f=\inf \{K:|f(y)-f(x)| \leqslant K d(y, x) \text { for all } y, x \in E\} .
$$

Let $L=\left\{f \in C_{0}\right.$ : Lip $\left.f<\infty\right\}$. If $P_{t}$ is a Feller semigroup, let Lip-exp $P_{t}$ (for Lipschitz exponent) be defined by

$$
\text { Lip-exp } P_{t}=\inf \left\{K: \operatorname{Lip} P_{t} f \leqslant e^{K t} \operatorname{Lip} f \text { for all } f \in L, \text { for all } t>0\right\} \text {. }
$$

Call a semigroup Lipschitz if Lip-exp $\mathrm{P}_{\mathrm{t}}<\infty$.

After proving a lemma, we show that if a semigroup is a combination of two Lipschitz semigroups, it will also be Lipschitz.

Let $D_{i} f$ be the $i$ th partial of $f$.

LEMMA 2.1. Suppose $P_{t}$ is a contraction semigroup such that if $f \in C_{0}^{2}$,

$$
\left\|P_{l} f-f\right\| \leqslant K t\left(\sum_{i=1}^{r}\left\|D_{i} f\right\|+\sum_{i, j=1}^{r}\left\|D_{i} D_{j} f\right\|\right) .
$$

If $P_{t}$ maps $C_{0}$ into bounded continuous functions, then $P_{t}$ maps $C_{0}$ into $C_{0}$.

Proof. Under the hypotheses given, for each $x$, there is a probability $P_{t}(x, \cdot)$ such that $P_{t} f(x)=\int f(y) P_{t}(x, d y)$ for all $f \in C_{0}$. Fix $x, N$. Let $g \in C_{0}^{2}$ such that $g(y)=4 N^{2}-(d(x, y))^{2}$ if $d(x, y) \leqslant N, 0 \leqslant g(y) \leqslant 3 N^{2}$ if $d(x, y)>N$, 
$\left\|D_{i} g\right\| \leqslant 4 N$ for each $i$, and $\left\|D_{i} D_{j} g\right\| \leqslant 4$ for each pair $i, j .\left|P_{t} g(x)-g(x)\right| \leqslant$ $K t\left(4 r N+4 r^{2}\right)$. So $P_{t}\left(x, S_{N}^{c}(x)\right) \leqslant 4 r^{2} K t(1+N) / N^{2}$, where $S_{N}(x)$ is the ball of radius $N$ about $x$.

If $f \in C_{0}, \varepsilon>0$, take $N$ large so that $4 r^{2} K t(1+N) / N^{2} \leqslant \varepsilon /\|f\|$. Then if $d(x,\{y:|f(y)|>\varepsilon\}) \geqslant N$,

$$
\left|P_{t} f(x)\right| \leqslant \varepsilon P_{t}\left(x, S_{N}(x)\right)+\|f\| P_{t}\left(x, S_{N}^{c}(x)\right) \leqslant 2 \varepsilon,
$$

which shows $P_{\imath} f \in C_{0}$.

For the remainder of this section we will suppose that all infinitesimal generators have domains that contain $C_{0}^{2}$, and that all generators satisfy

$$
\|A f\| \leqslant K_{A}\left(\sum_{i}\left\|D_{i} f\right\|+\sum_{i, j}\left\|D_{i} D_{j} f\right\|\right) \quad \text { if } f \in C_{0}^{2} \text {, for some constant } K_{A} .
$$

By Dynkin's formula, if $A$ generates $P_{t}, P_{t}$ will satisfy the hypotheses of 2.1 .

THEOREM 2.3. Let $P_{t}, Q_{t}$ be two Lipschitz semigroups with strong infinitesimal generators $A, B$, respectively. Suppose $C$ is the closure of the restriction of $A+B$ to $C_{0}^{2}$ and that $C$ is the strong infinitesimal generator of a contraction semigroup $R_{t}$. Then $R_{t}$ will be Lipschitz, and Lip-exp $R_{t} \leqslant \operatorname{Lip-exp} P_{t}+\operatorname{Lip}-\exp Q_{t}$.

Proof. By the Trotter product formula [6], if $f \in C_{0}^{2},\left(P_{t / n} Q_{t / n}\right)^{n} f \rightarrow R_{t} f$. Since $P_{s}, Q_{s}$ both map $C_{0}$ into $C_{0}$ for all $s, R_{t}$ maps $C_{0}^{2}$ into continuous functions and hence, by $2.1, C_{0}$ into $C_{0}$. Since $C_{0}^{2}$ is contained in the domain of $C, R_{t} f \rightarrow f$ uniformly as $t \rightarrow 0$ if $f \in C_{0}^{2}$, hence if $f \in C_{0}$. Thus $R_{t}$ is Feller.

If $f \in C_{0}^{2}$, and $K_{1}=$ Lip-exp $P_{t}, K_{2}=$ Lip-exp $Q_{t}$, then

$$
\operatorname{Lip}\left(P_{t / n} Q_{t / n}\right)^{n} f \leqslant\left(e^{K_{1} t / n} e^{K_{2} t / n}\right)^{n} \operatorname{Lip} f=e^{\left(K_{1}+K_{2}\right) t} \operatorname{Lip} f .
$$

It follows readily that $R_{t}$ is Lipschitz, and Lip-exp $R_{t} \leqslant K_{1}+K_{2}$.

Suppose $P_{t}$ is as above.

(2.4) Suppose $m(x, d y)$ is a nonnegative kernel that is uniformly bounded, weakly continuous in $x$, and there exists $M$ such that $m\left(x, S_{M}^{c}(x)\right)=0$ for all $x$, where $S_{M}(x)$ is the ball of radius $M$ about $x$.

If $B$ is the operator given by $B g(x)=\int[g(y)-g(x)] m(x, d y)$, it is known [2] that $B$ will generate a nonnegative contraction semigroup $Q_{t}$. By the conditions on $B, B: C_{0} \rightarrow C_{0}$. Since $B$ is bounded, one can write an explicit formula for $Q_{t}[10]$ and see that $Q_{t}$ maps $C_{0}$ into continuous functions. Note that

$$
\|B g\| \leqslant \sum_{i}\left\|D_{i} g\right\| \int\left(y_{i}-x_{i}\right) m(x, d y),
$$

where $x_{i}$ is the $i$ th coordinate of $x$, and so by $2.1, Q_{t}$ is Feller.

It is also known ([2] or [10]) that $A+B$ restricted to the domain of $A$ generates a nonnegative contraction semigroup $R_{t}$ on $C_{0}$. Thus, in this special case we do not have to suppose the existence of $R_{t}$, and we have

COROllary 2.5. Suppose $P_{t}$ is a Lipschitz semigroup with generator $A$. Let $m$ be $a$ kernel satisfying (2.4) and let $B$ be defined by $B g(x)=\int[g(y)-g(x)] m(x, d y)$. Then $A+B$ restricted to $C_{0}^{2}$ has an extension that generates a Feller semigroup $R_{t}$ and Lip-exp $R_{t} \leqslant$ Lip-exp $P_{t}+$ Lip-exp $Q_{t}$, where $Q_{t}$ is the semigroup generated by $B$. 
Proof. The only comment required is that if $Q_{t}$ is not Lipschitz, then Lip-exp $Q_{t}$ $=\infty$, and the inequality is trivial.

The next result says that if one has a sequence of uniformly Lipschitz semigroups, one can find a subsequence that converges.

THEOREM 2.6. Suppose $P_{t}^{n}$ is a sequence of Lipschitz semigroups with generators $A_{n}$. Suppose $K=\sup _{n}$ Lip-exp $P_{t}^{n}<\infty$, and $\sup _{n} K_{A_{n}}<\infty$, where $K_{A_{n}}$ is as in (2.2). Then there is a subsequence $P_{t}^{n_{j}}$ and a Lipschitz semigroup $P_{t}$ such that $P_{t}^{n_{1}} f \rightarrow P_{t} f$ uniformly whenever $f \in C_{0}$, and Lip-exp $P_{t} \leqslant K$.

Furthermore, if $\lim _{n \rightarrow \infty} A_{n} f=A f$ for $f \in C_{0}^{2}$ for some operator $A$, then $A$ restricted to $C_{0}^{2}$ has an extension which generates $P_{t}$.

Proof. If $f \in C_{0}^{2}$, then $P_{t}^{n} f$ is Lipschitz in $t$ with Lipschitz constant $c \sup _{n}\left\|A_{n} f\right\|$, where $c$ is independent of $n$. On the other hand, $P_{t}^{n} f$ is Lipschitz in $x$, and Lip $P_{t}^{n} f \leqslant e^{K t} \operatorname{Lip} f$. Thus $P_{t}^{n} f$ is equicontinuous in $x$ and $t$, and by Ascoli-Arzela, there is a subsequence that converges uniformly on compact sets in $[0, \infty) \times E$. The limit, call it $F$, will be continuous. By the argument of 2.1, if $d(x,\{y:|f(y)|>\varepsilon\}) \geqslant N$,

$$
\left|P_{t}^{n} f(x)\right| \leqslant \varepsilon+4 r^{2}\|f\| \sup _{n} K_{A_{n}} t(1+N) / N^{2},
$$

which, taking a limit along the subsequence, shows that $F \in C_{0}$. Also, $F$ will be Lipschitz in $x$ with Lipschitz constant no bigger than $e^{K t}$ Lip $f$.

Pick a countable dense subset of $C_{0}^{2}$. By a diagonalization process, one can find a subsequence $P_{t}^{n}$ that converges for each $f$ in this countable subset. Call the limit $P_{t} f$. Since this countable subset is dense in $C_{0}^{2}$, hence in $C_{0}$, extend $P_{t}$ to all of $C_{0}$, by continuity. Since each $P_{t}^{n}$ is a contraction, it is easy to check that $P_{t}$ is a nonnegative contraction semigroup that is Feller and that Lip-exp $P_{t} \leqslant K$.

Now suppose $A_{n} f \rightarrow A f$ uniformly if $f \in C_{0}^{2} . A_{n} f=\lim _{t \rightarrow 0}\left(P_{t}^{n} f-f\right) / t$ is continuous, and so $A f$ is continuous and bounded. By the hypotheses and the argument of 2.1, for fixed $x$ and $t$ the family of probabilities $P_{t}^{n}(x, \cdot)$ is tight. Since $P_{t}^{n_{j}} f(x) \rightarrow P_{t} f(x)$ whenever $f \in C_{0}, P_{t}^{n_{j}} A f(x) \rightarrow P_{t} A f(x)$. This holds for each $x$ and $t$. Similarly, for fixed $x$, the family $P_{t}(x, \cdot)$ is tight, and so $P_{t} A f(x) \rightarrow A f(x)$ as $t \rightarrow 0$.

Then if $f \in C_{0}^{2}$,

$$
\begin{aligned}
P_{t} f-f & =\lim _{j \rightarrow \infty}\left(P_{t}^{n_{j}} f-f\right)=\lim _{j} \int_{0}^{t} P_{s}^{n_{j}} A_{n_{j}} f d s \\
& =\lim _{j} \int_{0}^{t} P_{s}^{n_{j}}\left(A_{n_{j}} f-A f\right) d s+\lim _{j} \int_{0}^{t} P_{s}^{n_{j}} A f d s=\int_{0}^{t} P_{s} A f d s,
\end{aligned}
$$

the limit being a pointwise limit, and using the facts that $P_{s}^{n_{j}}$ is a contraction and $A_{n} f \rightarrow A f$ uniformly. Dividing both sides by $t$ and letting $t \rightarrow 0$ shows that $A$ restricted to $C_{0}^{2}$ is the restriction of the generator of $P_{t}$.

Let us say that a Lipschitz semigroup $Q_{t}$ is approximable if (i) there exist kernels $m_{n}$, each satisfying (2.4); (ii) if $Q_{t}^{n}$ is the semigroup generated by $B_{n}$ where $B_{n} g(x)=\int[g(y)-g(x)] m_{n}(x, d y)$, then Lip-exp $Q_{t}^{n} \leqslant$ Lip-exp $Q_{t}$; and (iii) $Q_{t}^{n} f \rightarrow$ $Q_{t} f$ uniformly as $n \rightarrow \infty$ whenever $f \in C_{0}$. 
Putting 2.6 and 2.5 together, we have

TheOREM 2.7. Suppose $P_{t}$ is a Lipschitz semigroup with generator $A$. Suppose $Q_{t}$ is a Lipschitz semigroup that is approximable such that if $B_{n}$ is the operator defined in (ii) of the definition of "approximable", $B_{n} f \rightarrow B f$ whenever $f \in C_{0}^{2}$, where $B$ is the infinitesimal generator of $Q_{t}$. Then $A+B$ restricted to $C_{0}^{2}$ has an extension that generates a Lipschitz semigroup $R_{t}$, and Lip-exp $R_{t} \leqslant \operatorname{Lip-exp} P_{t}+\operatorname{Lip}-\exp Q_{t}$.

Proof. Let $R_{t}^{n}$ be the semigroup generated by $A+B_{n}$. By 2.6 , there is a subsequence that converges to a semigroup; call it $R_{t}$.

$$
\begin{aligned}
\text { Lip-exp } R_{t} & \leqslant \sup _{n} \operatorname{Lip}-\exp R_{t}^{n} \leqslant \operatorname{Lip-exp} P_{t}+\sup _{n} \operatorname{Lip-exp} Q_{t}^{n} \\
& \leqslant \text { Lip-exp } P_{t}+\text { Lip-exp } Q_{t} .
\end{aligned}
$$

We need one final result.

THEOREM 2.8. Suppose for each $k, Q_{t}^{k}$ is approximable, $\sup _{k}$ Lip-exp $Q_{t}^{k}=M<$ $\infty$, and $Q_{t}^{k} \rightarrow Q_{t}$ for all $f \in C_{0}$. Then $Q_{t}$ is approximable.

Proof. Let the semigroups that approximate $Q_{t}^{k}$ be denoted by $Q_{t}^{k_{n}}$. Let $\left\{f_{i}\right\}$ be a countable dense subset of $C_{0}$. Let $\left\{\left(g_{j}, g_{j}^{k}, g_{j}^{k n}\right)\right\}$ be an ordering of $\left\{\left(Q_{r} f_{i}, Q_{r}^{k} f_{i}, Q_{r}^{k_{n}} f_{i}\right): r\right.$ rational $\}$.

There exists a $K_{1}$ such that if $k \geqslant K_{1},\left\|g_{1}-g_{1}^{k}\right\| \leqslant \frac{1}{2}$. For each $k \geqslant K_{1}$, there exists $N_{1}^{k}$ such that if $n \geqslant N_{1}^{k},\left\|g_{1}^{k n}-g_{1}^{k}\right\| \leqslant \frac{1}{2}$. There exists $K_{2}>K_{1}$ so that if $k \geqslant K_{2},\left\|g_{1}-g_{1}^{k}\right\| \leqslant \frac{1}{4},\left\|g_{2}-g_{2}^{k}\right\| \leqslant \frac{1}{4}$. There exists $N_{2}^{k}$ such that if $k \geqslant K_{2}$, $n \geqslant N_{2}^{k}, \quad\left\|g_{1}^{k n}-g_{1}^{k}\right\| \leqslant \frac{1}{4}, \quad\left\|g_{2}^{k n}-g_{2}^{k}\right\| \leqslant \frac{1}{4}$. Continue, defining $K_{p}$ so that $\left\|g_{j}-g_{j}^{k}\right\| \leqslant 2^{-p}, j=1,2, \ldots, p$, if $k \geqslant K_{p}$, and so on.

The sequence $Q_{r}^{K_{p}, N_{p}} f_{i}\left(=g_{j} K_{p}, N_{p}\right)$ will converge to $Q_{r} f_{i}\left(=g_{j}\right)$ for each $i$ and rational $r$. Since each $Q_{t}^{k n}$ is a contraction semigroup, we have $Q_{t}^{K_{p}, N_{p}}$ converges to $Q_{t} f$ for all $t \geqslant 0$, all $f \in C_{0}$.

3. Diffusions with Lipschitz coefficients. We now consider Markov processes on the real line ( $E=$ reals). The processes that we will consider will all contain $C_{0}^{2}$ in the domains of their infinitesimal generators. If $Z_{t}$ is the Markov process with generator $A f(x)=a^{2}(x) f^{\prime \prime}(x)$, where $a$ is a Lipschitz function, then using Ito's theory of stochastic differential equations (see [1]) it is relatively easy to show that the semigroup of $Z_{t}$ is Lipschitz.

THEOREM 3.1. Suppose $P_{t}$ is the semigroup generated by $A$, where $A f(x)=$ $a^{2}(x) f^{\prime \prime}(x),\|a\|<\infty$, and $\alpha=\operatorname{Lip} a<\infty$. Then $P_{t}$ is Lipschitz.

Proof. If $f \in C_{0}^{2},\left\|P_{t} f-f\right\|=\left\|\int_{0}^{t} P_{s} A f d s\right\| \leqslant\|a\|^{2} t\left\|f^{\prime \prime}\right\|$. By $2.1, P_{t}$ is Feller.

To show $P_{t}$ is Lipschitz, let $P$ be a probability under which $W_{t}$ is a standard Brownian motion, and let $X_{t}$ satisfy $X_{t}=x+\int_{0}^{t} a\left(X_{s}\right) d W_{s}$ and $Y_{t}$ satisfy $Y_{t}=y$ $+\int_{0}^{t} a\left(Y_{s}\right) d W_{s}$. 


$$
\begin{aligned}
E\left(X_{t}-Y_{t}\right)^{2}= & (x-y)^{2}+2(x-y) E \int_{0}^{t}\left[a\left(X_{s}\right)-a\left(Y_{s}\right)\right] d W_{s} \\
& +E\left[\int_{0}^{t}\left[a\left(X_{s}\right)-a\left(Y_{s}\right)\right] d W_{s}\right]^{2} \\
= & (x-y)^{2}+E \int_{0}^{t}\left[a\left(X_{s}\right)-a\left(Y_{s}\right)\right]^{2} d s \\
\leqslant & (x-y)^{2}+\alpha^{2} \int_{0}^{t} E\left(X_{s}-Y_{s}\right)^{2} d s .
\end{aligned}
$$

By Gronwall's inequality, $E\left(X_{t}-Y_{t}\right)^{2} \leqslant(x-y)^{2} e^{\alpha^{2} t}$. If $f \in L$,

$$
\begin{aligned}
\left|E f\left(X_{t}\right)-E f\left(Y_{t}\right)\right| & \leqslant(\operatorname{Lip} f) E\left|X_{t}-Y_{t}\right| \leqslant(\operatorname{Lip} f)\left(E\left(X_{t}-Y_{t}\right)^{2}\right)^{1 / 2} \\
& \leqslant(\operatorname{Lip} f)|x-y| e^{\alpha^{2} t / 2} .
\end{aligned}
$$

If $Z_{t}$ is the process with $A$ as its generator, then, as is well known, the distribution of $Z_{t}$ under $P^{x}$ is the same as $X_{t}$ under $P$ and the distribution of $Z_{t}$ under $P^{y}$ is the same as $Y$, under $P$. So,

$$
\begin{aligned}
\left|P_{t} f(x)-P_{t} f(y)\right| & =\left|E^{x} f\left(Z_{t}\right)-E^{y} f\left(Z_{t}\right)\right|=\left|E f\left(X_{t}\right)-E f\left(Y_{t}\right)\right| \\
& \leqslant \operatorname{Lip} f|x-y| e^{\alpha^{2} / 2},
\end{aligned}
$$

or Lip-exp $P_{t}=\alpha^{2} / 2$.

By a more careful analysis, however, we can do much better and show Lip-exp $P_{t}$ $=0$.

THEOREM 3.2. Under the same hypotheses as 3.1, Lip-exp $P_{t}=0$.

Proof. Take $h$ so that $\alpha h<1$; let $\lambda=2 / h^{2}$. Consider the Markov process $X_{t}^{h}$, with semigroup $P_{t}^{h}$, constructed as follows: starting at $x$, the process waits at $x$ a length of time that is exponential with parameter $\lambda$. At that time it jumps either to $x+a(x) h$ or $x-a(x) h$, both with probability $\frac{1}{2}(a(x)$ is possibly 0$)$. It waits at the new point a length of time that is exponential with parameter $\lambda$, and so on. It is easy to see that the generator of $P_{t}^{h}$ is given by

$$
A^{h} f(x)=[f(x+a(x) h)+f(x-a(x) h)-2 f(x)] / h^{2} .
$$

By 2.6, since $A^{h} f \rightarrow A f$ if $f \in C_{0}^{2}$, Lip-exp $P_{t}$ will be 0 if we show Lip-exp $P_{t}^{h}=0$. There is no question of subsequences here, since there is only one semigroup that has generator $A$.

Let $Q$ be the transition probability for the Markov chain that, starting at $x$, jumps either to $x+a(x) h$ or $x-a(x) h$, both with probability $\frac{1}{2}$. Let $Q^{n}$ be the iterates of $Q$. Since $X_{t}^{h}$ is a pure jump process with waiting times that are identically distributed,

$$
P_{t}^{h} f=e^{-\lambda t} \sum_{k=0}^{\infty} \frac{\left(Q^{k} f\right)(\lambda t)^{k}}{k !}
$$

If we show $\operatorname{Lip} Q^{k} f \leqslant \operatorname{Lip} f$, we would then have $\operatorname{Lip} P_{t}^{h} f \leqslant \operatorname{Lip} f$, our $\operatorname{desired}$ result.

$$
\left|Q^{k+1} f(y)-Q^{k+1} f(x)\right|=\left|Q\left(Q^{k} f\right)(y)-Q\left(Q^{k} f\right)(x)\right|
$$


If we show $\operatorname{Lip} Q f \leqslant \operatorname{Lip} f$ for all $f$, then since the case $k=0$ is trivial, we would do a simple induction and be done.

Suppose $y>x$.

$$
\begin{aligned}
|Q f(y)-Q f(x)|= & \mid \frac{1}{2} f(y+a(y) h)+\frac{1}{2} f(y-a(y) h) \\
& -\frac{1}{2} f(x+a(x) h)-\frac{1}{2} f(x-a(x) h) \mid \\
\leqslant & \frac{1}{2} \operatorname{Lip} f|(y-x)+(a(y)-a(x)) h| \\
& +\frac{1}{2} \operatorname{Lip} f(y-x)-(a(y)-a(x)) h \mid .
\end{aligned}
$$

Now $|(a(y)-a(x)) h| \leqslant \alpha h|y-x|<|y-x|$. Therefore,

$$
|(y-x)+(a(y)-a(x)) h|=(y-x)+(a(y)-a(x)) h
$$

and $|(y-x)-(a(y)-a(x)) h|=(y-x)-(a(y)-a(x)) h$. We thus have $|Q f(y)-Q f(x)| \leqslant \operatorname{Lip} f(y-x)$, which completes the proof.

4. Diffusions on the real line. Since Lip-exp $P_{t}=0$ under the conditions of 3.2 , regardless of $a$, any process that can be approximated by diffusions with Lipschitz coefficients will also have Lipschitz exponent 0 . We exploit this to show that a large class of diffusions on the line have Lipschitz exponent 0 . We consider those diffusions on $R$ which are on natural scale and whose speed measures $s$ satisfy

(4.1)(i) $s$ is finite on finite intervals and

(ii) there is a constant $\beta>0$ such that $s(x, y] \geqslant \beta(y-x)$ for all $x<y$.

The first condition insures that there are no boundary points, the second that the process does not move too fast.

Let $W_{t}$ be a standard Brownian motion and let $L_{t}^{y}$ be local time for $W$ at $y$. Recall that $L_{t}^{y}$ is jointly continuous in $t$ and $y$, increasing in $t$, that $\int_{A} L_{t}^{y} d y=$ $\int_{0}^{t} 1_{A}\left(W_{s}\right) d s$, and $E^{x}\left(L_{t}^{y}\right)^{2}$ as a function of $y$ is bounded on compact intervals. Recall that a diffusion on the real line which is on natural scale is characterized by its speed measure $s$. If $H_{t}=\int L_{t}^{y} s(d y), T_{t}=\inf \left\{u>0: H_{u} \geqslant t\right\}$, then $W_{T_{t}}$ will have the same distribution under $P^{x}$ as $X_{t}$, for all $x$. (See [4] or [9].)

Note that by (4.1)(ii), $H_{t}=\int L_{t}^{y} s(d y) \geqslant \beta \int L_{t}^{y} d y=\beta t$. So $T_{t}<t / \beta$, and if $f \in C_{0}^{2}$, by Dynkin's identity,

$$
E^{x} f\left(X_{t}\right)-f(x)=E^{x} f\left(W_{T_{t}}\right)-f(x)=E^{x} \int_{0}^{T_{t} \frac{1}{2}} f^{\prime \prime}\left(W_{s}\right) d s \leqslant(t / 2 \beta)\left\|f^{\prime \prime}\right\| .
$$

It is known that $P_{t}$ maps $C_{0}$ into continuous functions, and by $2.1, P_{t}: C_{0} \rightarrow C_{0}$. Furthermore, by the continuity of $L_{t}^{y}$ in $t, H_{t}$ is continuous.

Our method is to approximate $s$ by speed measures $s_{n}$ such that the corresponding processes $X_{t}^{n}$ with semigroups $P_{t}^{n}$ have generators $a_{n}^{2} f^{\prime \prime}$, where $a_{n}$ is Lipschitz, and to show that $P_{t}^{n}$ converges to $P_{t}$, the semigroup of $X_{t}$.

THeOREM 4.2. Suppose $X_{t}$ with semigroup $P_{t}$ is a diffusion on $R$ that is on natural scale and that its speed measure $s$ satisfies (4.1). Then Lip-exp $P_{t}=0$ and $P_{t}$ is approximable. 
Proof. First, suppose there exists $N$ such that $s$ on $[-N, N]^{c}$ is Lebesgue measure. We may assume $\beta \leqslant 1$ in (4.1)(ii). Define $S(x)=s(-N, x]$ for $-N \leqslant x \leqslant$ $N$, and let $S_{n}$ be a sequence of increasing $C^{2}$ functions on $[-N, N]$ that tend pointwise to $S$, except possibly at points of discontinuity of $S$, such that $S_{n}^{\prime}(x) \geqslant \beta$ for all $x, S_{n}(-N)=0$, and $S_{n}(N)=S(N)$. This is possible since $S$ is increasing and bounded. Let $s_{n}$ be the measure on $[-N, N]$ induced by $S_{n}$, and define $s_{n}$ on $[-N, N]^{c}$ to be Lebesgue measure on $[-N, N]^{c}$. Let $X_{t}^{n}$ be the diffusion which has $s_{n}$ as its speed measure, $P_{t}^{n}$ the corresponding semigroup, and $H_{t}^{n}$ and $T_{t}^{n}$ defined analogously to $H_{t}$ and $T_{t}$.

It is not hard to check that the generator of $P_{t}^{n}$ is of the form $a_{n}^{2} f^{\prime \prime}$, where $a_{n}$ is differentiable, bounded above by $\beta^{-1 / 2}$, and bounded below away from 0 (since $S_{n}^{\prime}$ is bounded above). By 3.2, Lip-exp $P_{t}^{n}=0$. Furthermore, the measures $s_{n}$ are uniformly bounded on finite intervals and if $g$ is continuous,

$$
\int_{[-N, N]} g(y) s_{n}(d y) \rightarrow \int_{[-N, N]} g(y) s(d y) .
$$

As before, $H_{u}^{n}-H_{t}^{n} \geqslant \beta(u-t), T_{t}^{n} \leqslant t / \beta$, and $P_{t}^{n} f(x)-f(x) \leqslant(t / 2 \beta)\left\|f^{\prime \prime}\right\|$.

Suppose $f \in C_{0}^{2}$. Then $\left\|P_{u}^{n} f-P_{t}^{n} f\right\| \leqslant\left\|P_{t}^{n}\right\|\left\|P_{u-t}^{n} f-f\right\| \leqslant(t-u)\left\|f^{\prime \prime}\right\| / 2 \beta$, or $P_{t}^{n}$ is Lipschitz in $t$.

Suppose now $f \in L$. Since $X_{t}$ has the same distribution as $W_{T_{t}}$ and $X_{t}^{n}$ has the same distribution as $W_{T_{t}^{n}}$,

$$
\begin{aligned}
\left|P_{t} f(x)-P_{t}^{n} f(x)\right| & =\left|E^{x} f\left(W_{T_{t}}\right)-E^{x} f\left(W_{T_{t}^{n}}\right)\right| \leqslant(\operatorname{Lip} f) E^{x}\left|W_{T_{t}}-W_{T_{t}^{n}}\right| \\
& \leqslant(\operatorname{Lip} f)\left(E^{x}\left(W_{T_{t}}-W_{T_{t}^{n}}\right)^{2}\right)^{1 / 2} .
\end{aligned}
$$

Since $W_{t}^{2}-t$ is a martingale and $\left(W_{T_{t}}-W_{T_{t}^{n}}\right)^{2}=\left(W_{T_{t} \vee T_{t}^{n}}-W_{T_{t} \wedge T_{t}^{n}}\right)^{2}$,

$$
E^{x}\left(W_{T_{t}}-W_{T_{t}^{n}}\right)^{2}=E^{x}\left|T_{t}-T_{t}^{n}\right| .
$$

Fix $\omega$. Suppose $T_{t}=u$. Then $H_{u}=t$. If $H_{u}^{n}=t-\varepsilon, H_{u+\varepsilon / \beta}^{n} \geqslant H_{u}^{n}+\beta(\varepsilon / \beta)=$ $t$. So $T_{t}^{u} \leqslant u+\varepsilon / \beta$. If $H_{u}^{n}=t+\varepsilon, H_{u-\varepsilon / \beta}^{n} \leqslant H_{u}^{n}-\varepsilon=t$, and $T_{t}^{n} \geqslant u-\varepsilon / \beta$. Thus $\left|T_{t}-T_{t}^{n}\right| \leqslant\left|H_{T_{t}}-H_{T_{t}}^{n}\right| / \beta$. We have then

$$
E^{x}\left|T_{t}-T_{t}^{n}\right| \leqslant E^{x}\left|H_{T_{t}}-H_{T_{t}^{n}}\right| / \beta .
$$

Let $h(y)=1$ if $|y| \leqslant n, N+1-|y|$ if $N \leqslant|y| \leqslant N+1$, and 0 otherwise. By the construction of $s_{n}$ and $s$,

$$
G_{n}=\left|H_{T_{t}}-H_{T_{t}}^{n}\right|=\left|\int h(y) L_{T_{t}}^{y} s(d y)-\int h(y) L_{T_{t}}^{y} s_{n}(d y)\right| .
$$

For each $\omega, h(y) L_{T_{t}}^{y}(\omega)$ as a function of $y$ is continuous with compact support, and so $G_{n}(\omega) \rightarrow 0$. On the other hand,

$$
\begin{aligned}
\sup _{n} E^{x}\left(\int h(y) L_{T_{t}}^{y} s_{n}(d y)\right)^{2} & \leqslant \sup _{n} \int h^{2}(y) E^{x}\left(L_{T_{t}}^{y}\right)^{2} s_{n}(d y) \\
& \leqslant \sup _{n} \int h^{2}(y) E^{x}\left(L_{t / \beta}^{y}\right)^{2} s_{n}(d y) \\
& \leqslant \sup _{|y|<N+1} E^{x}\left(L_{t / \beta}^{y}\right)^{2} \sup _{n} s_{n}[-N-1, N+1]<\infty .
\end{aligned}
$$


A similar equation holds for $E^{x}\left(\int h(y) L_{T_{t}}^{y} s(d y)\right)^{2}$; hence $\sup _{n} E_{x}^{x} G_{n}^{2}<\infty$, or the $G_{n}$ are uniformly integrable, and so $E^{x} G_{n} \rightarrow 0$.

Thus, $P_{t}^{n} f(x) \rightarrow P_{t} f(x)$ for each $x$ if $f \in L$. Since $P_{t}^{n} f$ is equicontinuous if $f \in L$, this, 2.6, and 2.1, gives uniform convergence. Hence Lip-exp $P_{t}=0$.

Finally, suppose $s$ satisfies (4.1). Define $s_{N}$ to be equal to $s$ on $[-N, N]$ and equal to Lebesgue measure on $[-N, N]^{c}$, and let $P_{t}, P_{t}^{N}, X_{t}, X_{t}^{N}$ be the corresponding semigroups and processes.

If $x$ is fixed and $T_{N}=\inf \left\{t>0:\left|X_{t}-x\right| \geqslant N\right\}, X_{t}$ and $X_{t}^{N}$ may be constructed as time changes of the same Brownian motion so that they have the same paths up to and including time $T_{N}$. If $g$ is the function constructed in the proof of 2.1,

$$
\left|E^{x} g\left(X_{T_{N} \wedge t}^{N}\right)-g(x)\right| \leqslant\left|E^{x} \int_{0}^{T_{N} \wedge t} \frac{4}{\beta} d s\right| \leqslant 4 \frac{t}{\beta},
$$

or $P^{x}\left(t \geqslant T_{N}\right) \leqslant 4 t /\left(\beta N^{2}\right)$. The same holds for $X_{t}$, and so if $f \in C_{0}$,

$$
\left|P_{t} f(x)-P_{t}^{N} f(x)\right| \leqslant 2\|f\| P^{x}\left(t \geqslant T_{N}\right) \leqslant 8 t\|f\| /\left(\beta N^{2}\right) \rightarrow 0
$$

as $N \rightarrow \infty$. Thus if $f \in L, P_{t}^{N} f \rightarrow P_{t} f$ pointwise, hence uniformly on intervals since $P_{t}^{N} f$ is equicontinuous, hence uniformly by 2.1 . Therefore Lip-exp $P_{t}=0$ and $P_{t}$ is approximable.

5. Drift. So far we have considered diffusions on the line that are on natural scale, that is, they have no drift. Now we consider adding drift terms.

Theorem 5.1. If Lip $b<\infty, A$ is the operator defined by $A f(x)=b(x) f^{\prime}(x)$ for $f \in C_{0}^{2}$, and $P_{t}$ is the semigroup generated by $A$, then $P_{t}$ is approximable and Lip-exp $P_{t} \leqslant \operatorname{Lip} b$.

Proof. Let $X_{t}^{h}$ be the process that starting at $x$, waits at $x$ a length of time that is exponential with parameter $1 / h$, then jumps to $x+b(x) h$, waits there a length of time that is exponential with parameter $1 / h$, and so on. Let $Q$ be the transition probability that at each step jumps from $x$ to $x+b(x) h, Q^{n}$ the iterates. Then exactly as in the proof of 3.2 ,

$$
\operatorname{Lip} P_{t}^{h} f \leqslant e^{-t / h} \sum_{k=0}^{\infty} \frac{\left(\operatorname{Lip} Q^{k} f\right)(t / h)^{k}}{k !},
$$

where $P_{t}^{h}$ is the semigroup for $X_{t}^{h}$.

$$
|Q f(y)-Q f(x)|=|y+b(y) h-(x+b(x) h)| \leqslant|y-x|(1+(\operatorname{Lip} b) h) .
$$

By induction, Lip $Q^{k} f \leqslant(1+h \operatorname{Lip} b)^{k}$, and so

$$
\operatorname{Lip} P_{t}^{h} \leqslant e^{-t / h} e^{t / h(1+h \operatorname{Lip} b)}=e^{t \operatorname{Lip} b} .
$$

Now let $h \rightarrow 0$ and use 2.6 .

6. Jumps. Ito's method of solving stochastic differential equations has been extended by Skorokhod [16] and Lepeltier and Marchal [14] to include the case where jumps are allowed. Thus, they consider solutions to the equation $X_{t}=x+$ $\int_{0}^{t} \int F\left(X_{s}, u\right) q(d s, d u)$ where $p(d s, d u)$ is the random measure associated to a 
certain process with stationary, independent increments, $m(d u)=E\left(\int_{0}^{1} p(d s, d u)\right)$, $q(d s, d u)=p(d s, d u)-m(d u) d s$, and $F$ satisfies $\int[F(y, u)-F(x, u)]^{2} m(d u)<$ $K|y-x|^{2}$. In a way very similar to 3.1 , we can show that the semigroup $P_{t}$ corresponding to $X_{t}$ is Lipschitz and Lip-exp $P_{t}$ is some function of $K$. Again, a more careful analysis shows that in some cases Lip-exp $P_{t}=0$.

Proposition 6.1. Let $m(x, d y)$ be a kernel on the real line such that:

(i) $m$ is weakly continuous in $x$;

(ii) $m(x, \cdot)$ puts positive mass $a_{i}$ on each of $n$ points $x+h_{i}(x)$, where $h_{i}(x)$ may possibly be 0 ;

(iii) $\sum_{i=1}^{n} a_{i} h_{i}(x)=0$ for all $x$;

(iv) $\sup _{i}\left\|h_{i}\right\|<\infty$ and Lip $h_{i}<1$ for all $i$.

Define A by

$$
\operatorname{Ag}(x)=\int[g(y)-g(x)] m(x, d y)
$$

and let $X_{t}, P_{t}$ be the process and semigroup generated by $A$. Then $P_{t}$ is Lipschitz and Lip-exp $P_{t}=0$.

Proof. Note that by (iii), $\int(y-x) m(x, d y)=0$, and so if $g \in C_{0}^{2}$,

$$
A g(x)=\int\left[g(y)-g(x)-g^{\prime}(x)(y-x)\right] m(x, d y) .
$$

It follows that whenever $A$ is given by (6.3) $\|A g\| \leqslant\left\|g^{\prime \prime}\right\|\left\|\int(y-x)^{2} m(x, d y)\right\|$ and, using 2.1, $P_{t}$ is Feller.

Let $M=\sum_{i=1}^{n} a_{i}$. Let $Q$ be the Markov chain that moves from $x$ to $x+h_{i}(x)$ with probability $a_{i} / M$. It is not hard to check that $X_{t}$ is the process that waits at $x$ a length of time that is exponential with parameter $M$, moves according to $Q$, then waits again, etc. Suppose $y>x$.

$$
\begin{aligned}
|Q f(y)-Q f(x)| & =\left|\sum a_{i} M^{-1} f\left(y+h_{i}(y)\right)-\sum a_{i} M^{-1} f\left(x+h_{i}(x)\right)\right| \\
& \leqslant(\operatorname{Lip} f) M^{-1} \sum a_{i}\left|y+h_{i}(y)-\left(x+h_{i}(x)\right)\right| .
\end{aligned}
$$

Since Lip $h_{i}<1,\left|h_{i}(y)-h_{i}(x)\right|<|y-x|$, so

$$
\left|y+h_{i}(y)-x-h_{i}(x)\right|=y-x+h_{i}(y)-h_{i}(x) .
$$

Then

Thus Lip $Q f \leqslant \operatorname{Lip} f$.

$$
\begin{aligned}
\| Q f(y)-Q f(x) \mid \leqslant & (\operatorname{Lip} f) M^{-1} \sum a_{i}(y-x) \\
& +(\operatorname{Lip} f) M^{-1}\left(\sum a_{i} h_{i}(y)-\sum a_{i} h_{i}(x)\right) \\
= & (\operatorname{Lip} f)|y-x| .
\end{aligned}
$$

As in 3.2, $\operatorname{Lip} Q^{k} f \leqslant \operatorname{Lip} f$,

$$
\text { Lip } P_{t} f \leqslant e^{-M t} \sum_{k=0}^{\infty} \frac{\operatorname{Lip} Q^{k} f(M t)^{k}}{k !} \leqslant \operatorname{Lip} f
$$

or Lip-exp $P_{t}=0$. 
Proposition 6.4. Suppose $m_{n}$ is a sequence of kernels such that (i) $\sup _{n}\left\|m_{n}(x, E)\right\|$ $<\infty$, (ii) $\sup _{n}\left\|\int|y-x| m_{n}(x, d y)\right\|<\infty$, and (iii) if $A_{n}$ is defined in terms of $m_{n}$ by (6.2) and $P_{t}^{n}$ is the semigroup generated by $A_{n}$, then each $P_{t}^{n}$ is Lipschitz and $\sup _{n}$ Lip-exp $P_{n}^{n}<\infty$. Suppose $m_{n}(x, d y)$ converges weakly to $m(x, d y)$ as $n \rightarrow \infty$ for each $x, A$ is defined in terms of $m$ by (6.2), and $P_{t}$ is the semigroup generated by $A$. Then $P_{t}$ is approximable, Lipschitz, and Lip-exp $P_{t} \leqslant \sup _{n}$ Lip-exp $P_{t}^{n}$.

Proof. If $f \in C_{0}^{2}$, note that

$$
\begin{aligned}
\left\|P_{t}^{n} f-f\right\| & \leqslant t\|A f\| \leqslant t\left\|f^{\prime}\right\| \int|y-x| m(x, d y) \\
& \leqslant t\left\|f^{\prime}\right\| \sup _{n}\left\|\int|y-x| m_{n}(x, d y)\right\| .
\end{aligned}
$$

By 2.6, there is a subsequence $n_{j}$ and a semigroup $Q_{t}$ such that $P_{t}^{n_{j} f} \rightarrow Q_{t} f$ uniformly whenever $f \in C_{0}^{2}$.

If $f \in C_{0}^{2}$,

$$
\begin{aligned}
Q_{t} f-f & =\lim _{j=\infty}\left(P_{t}^{n_{j}} f-f\right)=\lim \int_{0}^{t} A_{n_{j}} P_{r}^{n_{j}} f d r \\
& =\lim \int_{0}^{t} A_{n_{j}}\left(P_{r}^{n_{j}} f-Q_{r} f\right) d r+\lim \int_{0}^{t} A_{n_{j}} Q_{r} f d r .
\end{aligned}
$$

The first limit is 0 since the $A_{n}$ 's are uniformly bounded. The second limit is $\int_{0}^{t} A Q_{r} f(x) d r$ for each $x$, since $Q_{r} f$ is bounded and continuous and $m_{n}$ converges weakly to $m$. Since $A$ is bounded, dividing both sides by $t$ gives $\lim _{t \rightarrow 0}\left(Q_{t} f-f\right) / t$ $=A f$, uniformly in $x$. Therefore

$$
Q_{t} f-P_{t} f=\int_{0}^{t} A\left(Q_{r} f-P_{r} f\right) d r \leqslant\|A\| \int_{0}^{t}\left(Q_{r} f-P_{r} f\right) d r
$$

or $Q_{t} f=P_{t} f$ if $f \in C_{0}^{2}$, hence for all $f \in C_{0}$.

Since any convergent subsequence of $P_{t}^{n}$ converges to $P_{t}, P_{t}^{n}$ converges to $P_{t}$.

We use 6.1 and 6.4 to get

THEOREM 6.5. Let $\mu$ be a continuous measure with $\int u^{2} \mu(d u)<\infty$. Let $F$ satisfy $\left\|\int|F(x, u)|^{2} \mu(d u)\right\|<\infty, \quad\left\|\int_{|u| \leqslant \varepsilon}|F(x, u)|^{2} \mu(d u)\right\| \rightarrow 0$ as $\varepsilon \rightarrow 0$, and $\mid F(x, u)-$ $F(y, u)|\leqslant| x-y \mid$ for all $x, y$. Let $m$ be the kernel defined by

$$
m(x, B)=\int 1_{B-x}(F(x, u)) \mu(d u)
$$

Let $A$ be defined by (6.3). Then $A$ will be the generator of an approximable semigroup with Lipschitz exponent 0.

Comment. Since $m(d u)$ is on the order of $o\left(u^{-2}\right) d u$, the condition of [9], $\int[F(x, u)-F(y, u)]^{2} m(d u) \leqslant K|x-y|^{2}$, translates, very roughly, to $\mid F(x, u)-$ $F(y, u) \mid$ is on the order of $u|x-y|$, a stronger requirement on $\operatorname{Lip} F(\cdot, u)$ than in 6.5 . 
Proof. If $\mu_{\varepsilon}(d u)=1_{[\varepsilon, \infty)}(|u|) \mu(d u), m_{\varepsilon}$ and $A_{\varepsilon}$ are defined analogously to $m$ and $A$, and $g \in C_{0}^{2}$,

$$
\begin{aligned}
A g-A_{\varepsilon} g & \leqslant\left\|g^{\prime \prime}\right\|\left\|\int(y-x)^{2}\left(m(x, d y)-m_{\varepsilon}(x, d y)\right)\right\| \\
& =\left\|g^{\prime \prime}\right\|\left\|\int_{|u|<\varepsilon}|F(x, u)|^{2} \mu(d u)\right\| \rightarrow 0
\end{aligned}
$$

By 2.6 , if the semigroup generated by $A_{\varepsilon}$ has Lipschitz exponent 0 , we will be done. Thus it suffices to assume $\mu$ is a bounded measure.

Next we approximate the drift by jumps. Let $h(x)=\int(y-x) m(x, d y)$. Let $u_{0}$ be fixed, let $\varepsilon_{x}(d y)$ denote point mass at $x$, and let $\mu_{n}=\mu+n \varepsilon_{u_{0}}$. Redefine $F\left(x, u_{0}\right)$ to be $h(x) / n$. If $n$ is sufficiently large, $\left|F\left(x, u_{0}\right)-F\left(y, u_{0}\right)\right| \leqslant|x-y|$. Define $m_{n}$ in terms of $\mu_{n}$ by (6.6) and define $A_{n}$ in terms of $m_{n}$ by (6.2). One can now check that $A_{n} g \rightarrow A g$ if $g \in C_{0}^{2}$, and so by 2.6 , it suffices to consider the case when $\mu$ is bounded and $A$ is given by (6.2).

Finally, let $\mu_{k}$ be a sequence of uniformly bounded measures that each put mass on at most $k$ points so that $\mu_{k}\left(\left\{u_{0}\right\}\right)=\mu\left(\left\{\mu_{0}\right\}\right)$ for each $k, \mu_{k} \rightarrow \mu$ weakly, and $\sup _{k}\left\|\int|F(x, u)|^{2} \mu_{k}(d u)\right\|<\infty$. If the $m_{k}$ are defined by (6.6) the $m_{k}(x, d y)$ will then converge weakly to $m(x, d y)$ for each $x$. Note that

$$
\begin{aligned}
\int|y-x| m_{k}(x, d y) & =\int|F(x, u)| \mu_{k}(d u) \\
& \leqslant \sup _{k}\left(\left\|\int|F(x, u)|^{2} \mu_{k}(d u)\right\|+\mu_{k}(E)\right) .
\end{aligned}
$$

Using 6.4 it suffices to consider the case where $A$ is given by (6.2) and $m$ satisfies (6.1)(i)-(iv). But then 6.1 gives the desired result.

7. Other jump processes. There are a number of jump processes that have Lipschitz semigroups, but which do not fit into the Skorokhod framework. For example, let $A$ be given by $A g(x)=a(x)[g(x+1)-g(x)]$, where Lip $a<\infty$. This is the process that waits a length of time that is exponential with parameter $1 / a(x)$, then jumps 1. Under the Skorokhod framework, the $F$ could not even be continuous, let alone Lipschitz.

Proposition 7.1. Let $A$ be given by $A g(x)=a(x)[g(x+h)-g(x)],\|a\|<\infty$. If $P_{t}$ is the semigroup generated by $A$, Lip-exp $P_{t} \leqslant h$ Lip $a$.

Proof. If $\|\mathrm{a}\|=0$ or Lip $a=\infty$, the result is trivial. So assume $\|a\|>0$ and Lip $a<\infty$. Let $M=\|a\|, b(x)=a(x) / M$.

Let $Q$ be the transition operator for the chain that goes from $x$ to $x+h$ with probability $b(x)$, and stays at $x$ with probability $1-b(x)$. Let $X_{t}$ be the process that jumps according to $Q$ and in between waits lengths of time that are exponential with parameter $M$. 
It is not hard to check that $X_{t}$ has generator $A$.

$$
\begin{aligned}
\mid Q f(y)- & Q f(x) \mid \\
= & |b(y) f(y+h)+(1-b(y)) f(y)-b(x) f(x+h)-(1-b(x)) f(x)| \\
\leqslant & b(x)|f(y+h)-f(x+h)|+[1-b(x)]|f(y)-f(x)| \\
& \quad+|b(y)-b(x)||f(y+h)-f(y)| \\
\leqslant & \operatorname{Lip} f|y-x|+(\operatorname{Lip} b)|y-x|(\operatorname{Lip} f) h \\
= & \operatorname{Lip} f|y-x|(1+h \operatorname{Lip} b) .
\end{aligned}
$$

If $P_{t}$ is the semigroup corresponding to $X_{t}, P_{t}=e^{-M t} \Sigma Q^{k}(M t)^{k} / k !$, or Lip $P_{t} \leqslant$ $\exp (M t h \operatorname{Lip} b)$, or Lip-exp $P_{t} \leqslant M h \operatorname{Lip} b=h \operatorname{Lip} a$.

We can then get the following theorem:

THEOREM 7.2. Suppose $A$ is given by (6.3), where $m$ is any kernel that satisfies (i) $\int(y-x)^{2} m(x, d y)$ is uniformly bounded; (ii) $\int_{|y-x|>\varepsilon}(y-x) m(x, d y) \rightarrow 0$ uniformly; and (iii) there is a number $M>0$ such that for any interval $[\alpha, \beta]$ that does not contain $0, \operatorname{Lip}\left(\int_{\alpha+x}^{\beta+x}(y-x) m(x, d y)\right) \leqslant M$. Then the semigroup generated by $A$ will be Lipschitz.

Proof. If we define $m_{\varepsilon}(x, d y)=1_{S_{\varepsilon}^{c}(x)}(y) m(x, d y)$, and define $A_{\varepsilon}$ in terms of $m_{\varepsilon}$ by (6.3), then just as in the proof of 6.5 , we can show that we need only consider the case where $m$ is uniformly bounded.

If we let $h(x)=\int(y-x) m(x, d y)$, and $a(x)=h(x) /\|h\|$, define $m_{n}(x, d y)=$ $m(x, d y)+n h(x) \varepsilon_{x+1 / n}(d y)$ if $h$ is positive, $m_{n}(x, d y)=m(x, d y)+$ $n h(x) \varepsilon_{x-1 / n}(d y)$ if $h$ is negative. If we define $A_{n}$ in terms of $m_{n}$ by (6.2), then $A_{n} g \rightarrow A g$ whenever $g \in C_{0}^{2}$, and so by 2.6 , we need only consider the case where $m$ is uniformly bounded, and $A$ is given by (6.2).

Finally, approximate $m$ by $m_{k}$ where

$$
m_{k}(x, d y)=\sum_{j=-k^{2}}^{k^{2}} \varepsilon_{j / k}(d y) m(x,[j / k,(j+1) / k)) .
$$

By 6.4, if the semigroups generated by $A_{k}$, where the $A_{k}$ are defined by (6.2), have a uniformly bounded Lipschitz exponent, we will have our result. That they do follows from hypothesis (iii), 7.1, and 2.7.

Consider operators that are of the form described in either 4.2, 5.1, 6.5, or 7.2. If $A$ is the sum of such operators, then by $2.7 A$ restricted to $C_{0}^{2}$ will have an extension that generates a Lipschitz semigroup.

We give an example to show that not every semigroup is Lipschitz. Let $P_{t}$ be the semigroup whose infinitesimal generator $A$ is given by $A f(x)=f(x+1)-f(x)$ if $x \geqslant 0, f(x-1)-f(x)$ if $x<0$. The Markov process corresponding to $P_{t}$ behaves like the standard Poisson process starting at $x$ if $x \geqslant 0$ and behaves like the negative of the Poisson process starting at $x$ if $x<0$. If $f(x)=x /\left(1+|x|^{2}\right)$ and $t>0$, it is easy to check that $\inf _{x \geqslant 0} P_{t} f(x)>0$ and $\sup _{x<0} P_{t} f(x)<0$. Thus $P_{t} f$ is not even continuous at 0 , let alone Lipschitz there. 


\section{REFERENCES}

1. L. Arnold, Stochastic differential equations: Theory and applications, Wiley, New York, 1974.

2. R. Bass, Adding and subtracting jumps from Markov processes, Trans. Amer. Math. Soc. 255 (1979), 363-376.

3. R. Blumenthal and R. Getoor, Markov processes and potential theory, Academic Press, New York, 1968.

4. L. Breiman, Probability, Addison-Wesley, Reading, Mass., 1968.

5. H. Brezis, W. Rosenkrantz and B. Singer, On a degenerate elliptic-parabolic equation occurring in the theory of probability, Comm. Pure Appl. Math. 24 (1971), 395-416.

6. P. Chernoff, Note on product formulas for operator semigroups, J. Funct. Anal. 2 (1968), 238-242.

7. G. Fichera, Sulle equazioni differenziali lineari ellittico-paraboliche del secondo ordine, Atti Accad. Naz. Lincei Mem. Ser. (8) 5 (1956), 1-30.

8. M. I. Freidlin, A priori estimates of solutions of degenerate elliptic equations, Dokl. Akad. Nauk SSSR 158 (1964), 281-283 = Soviet Math. Dokl. 5 (1964), 1231-1234.

9. K. Ito and H. P. McKean, Jr., Diffusion processes and their sample paths, Academic Press, New York, 1965.

10. T. Kato, Perturbation theory for linear operators, Springer-Verlag, New York, 1966.

11. J. J. Kohn and L. Nirenberg, Degenerate elliptic-parabolic equations of second order, Comm. Pure Appl. Math. 20 (1967), 797-872.

12. T. Komatsu, Markov processes associated with certain integro-differential operators, Osaka J. Math. 10 (1973), 271-303.

13. V. A. Kostin, Smoothness of solutions of certain parabolic equations. I, II, III, Differential Equations 12 (1976), 1054-1063; ibid. 12 (1976), 1139-1143; ibid. 12 (1976), 1470-1473.

14. J. P. Lepeltier and B. Marchal, Probleme des martingales et equations différentielles stochastiques associées à un opérateur intégro-différentiel, Ann. Inst. H. Poincaré 12 (1976), 43-103.

15. O. A. Oleinik, On the smoothness of solutions of degenerate elliptic and parabolic equations, Dokl. Akad. Nauk SSSR 163 (1965), 577-580 = Soviet Math. Dokl. 6 (1965), 972-976.

16. A. Skorokhod, Studies in the theory of random processes, Addison-Wesley, Reading, Mass., 1965.

17. D. Stroock, Diffusion processes associated with Lévy generators, Z. Wahrsch. Verw. Gebiete 32 (1975), 209-244.

Department of Mathematics, University of Washington, Seattle, Washington 98195 